\title{
ESTIMATING THE MONETARY BENEFITS OF TELEREHABILITATION IN THE COUNTRYSIDE - CASE OF INTRODUCTION OF TELE-HOMECARE
}

\author{
Päivi Kekkonen ${ }^{1}$, Arto Reiman ${ }^{1}$, Pirjo Lappalainen ${ }^{2}$, Marika Tuiskunen ${ }^{2}$ and Kirsi Jokinen ${ }^{2}$ \\ ${ }^{1}$ Industrial Engineering and Management, University of Oulu, P.O. Box 4610, 90014 University of Oulu, Finland \\ ${ }^{2}$ Social Services and Rehabilitation, Oulu University of Applied Sciences, P.O. Box 222, 90101 Oulu, Finland
}

\begin{abstract}
Digitalization provides new solutions for health and welfare services. In cases where the customer has a long distance to reach the face-to-face services or has other limitations related to mobility, telerehabilitation can be used to provide the needed services for the customer. This study presents a tele-homecare service introduced in a municipal health and welfare services provider for the elderly. The cost saving potential of tele-homecare service as compared to traditional homecare service is a key interest. A categorization of costs in cases of traditional and tele-homecare is presented and further effects of tele-homecare in terms of costs are discussed. The case study is a part of an on-going project, the aim of which is to provide information that can be utilized in making well-grounded decisions on introducing telerehabilitation services that take into account the special needs and characteristics of rural areas.
\end{abstract}

\section{KEYWORDS}

Demographic Change, Cost Accounting, Digitalization of Services, Tele-Homecare, Telerehabilitation

\section{INTRODUCTION}

Global megatrends, such as digitalization, aging population and the current economic situation throughout Europe have forced the nations to develop cost-efficient solutions and practices in healthcare. Different telemedicine practices have been introduced through the development of information and communication technologies (ICT) during the 2000s', yet until recent years the mobilization of such solutions has been rather modest. (Dorsey \& Topol, 2020) However, the utilization of ICT in facilitating remote work has expanded in 2020 due to the global crisis as a consequence to the COVID -19 virus (see e.g. Heymann and Shindo, 2020; Hollander and Carr, 2020).

In this study we focus telerehabilitation in the countryside in northern Finland. Our case studies on telerehabilitation are performed in sparsely populated areas where long distances and aging population are typical. In practice, our target groups fit well to a more general definition by Selzler et al (2018) who bring out in their review that there are people living in such rural areas who need rehabilitation services but who have either physical or social limitations, or both, related to mobility. In our cases, examples of such people are elderly living on their own, disabled people, and others, both young and old, who are at risk of exclusion socially and from the job market. Both practitioners and decision makers at our focus areas have expressed the need for low threshold rehabilitation services among these target groups.

Telerehabilitation means providing rehabilitation with the use of different telecommunication networks and ICT solutions. Telerehabilitation that can be called or referred as to e-rehabilitation, virtual rehabilitation, telehealth, telepractice or teletreatment is meant to facilitate rehabilitation through technology (Tousignant et al., 2015). Telerehabilitation is a process that provides equal access to the necessary services for the inhabitants of sparsely populated areas, thus responding not only to the social and health care needs, but also on those related to wider societal perspective (Tousignant et al., 2015).

In our ongoing research and development project, the aim is to introduce new telerehabilitation practices in a municipal target organization and test these practices in real-life rehabilitation contexts (Kekkonen et al. 2019). Telerehabilitation practices are examined from the viewpoints of an individual taking part in the 
rehabilitation, the rehabilitation service provider and their employees, and the municipalities responsible for offering rehabilitation services. Testing of new telerehabilitation practices include an examination focusing on their costs and benefits on monetary terms, contributing thus to an ongoing scientific debate on the economic aspects related to healthcare (Hill, 2010, Tousignant et al., 2015). Our economic analysis is based on the cost-benefit analysis method presented by Oxenburgh and Marlow (2005) and in this article we present the categorization of the costs related to telerehabilitation and calculate different scenarios for those cost elements in which we consider variation exists.

The article continues with a presentation of a study setting involving tele-homecare service. The results section consists of a calculation comparing the costs of the tele-homecare and traditional homecare. The approximations used in the calculation, its validity and limitations of the study are presented in the discussion section together with future research interests on the theme. In the conclusions the results are wrapped up with notions related to the wider perspective of the role and increase of tele-healthcare solutions in the future.

\section{STUDY SETTING}

Target organization of this study is a municipal health and welfare services provider for the elderly. The organization operates in North-Eastern Finland and offers services to a population of approximately 15000. According to an internal population forecast by the municipality the amount of the elderly people (aged 75 years and above) in the population of the municipality is estimated to increase from the current approximately 1900 individuals to 2700 by the year 2030 and further to 3200 by the year 2040. Characteristic to the area are also long distances and a minor share of the population living in remote locations of up to 60 kilometers from the center of the municipality.

In this study, tele-homecare services provided by the target organization are examined as an example of introducing remote health and welfare service for the elderly. In the year 2018 homecare services were provided for 240 customers in total. The introduction of tele-homecare services was initiated in the target organization in 2019. The data used in the following calculations is based on an interview carried out with the homecare service manager of the target organization and further updates carried out by e-mail concerning the figures used for calculation. Approximations by the researchers are used to supplement the data provided the target organization as described in the following section.

\section{RESULTS}

A calculation of costs in cases of traditional and tele-homecare services is presented in Table 1. The table includes columns for costs targeted for both the municipal service provider and the customer. The rows include different categories of costs identified earlier in this project together with the representatives of target organizations (Kekkonen et al. 2019). The calculation is carried out with following estimates:

The service in the target organization was initiated by recruiting and training three employees to provide the tele-homecare service. If their daily work time is not filled by tele-home visits, they also do traditional homecare work. In daily work the actual work time of traditional and tele-homecare visits was estimated to be the same and thus the direct work costs similar. The tele-homecare was not viewed to call for more planning or preparation on behalf of the employee and this also does not produce a difference between the work costs of traditional and tele-mediated method.

In the calculation of costs in following categories, an amount of 25 customers was used. This amount of customers was estimated to fill the daily work time of tele-homecare service employee. The Daily amount of visits for the traditional home-care used in the calculation is three visits. The amount of 25 customers could not be cared for with the same employee work costs in traditional home care with this amount of visits, although this is assumed in this calculation. Three daily visits for each of the 25 customers in the traditional homecare during one month would add up to 2250 visits in total.

The biggest difference, in terms of the costs between traditional and tele homecare, is the travel costs. These are highly affected by the location of the homes of the customers. In case of our target organization, the distance between the customer and the homecare services can span between $0 \mathrm{~km}$ to $60 \mathrm{~km}$. Here an average distance of 1 kilometer for the home of the customer was used, since most of the customers live in 
the center of the municipality. A two-way trip to the home of the customer and back to the workplace was assumed, although in reality the routing is something else based on the locations of the customers. With a short average distance this should not contribute to a major error. The travel costs are dependent on the transportation method of the employee. In this case the travel costs were calculated using the current national mileage allowance. In addition to actual cost of travelling, also the work time used for the travelling needs to be considered. This was estimated by using the average travel speed corresponding to city area $(40 \mathrm{~km} / \mathrm{h})$ for the short distances used in the calculation. An additional time of 6 minutes for the transitions from/to the vehicle to/from inside of the building for each travel leg was added.

In terms of customer fees the target organization had made a decision of offering the tele-homecare service for the customers for a price that is $10 \%$ lower than the traditional homecare service customer fee. The actual individual customer fee is determined based on the income of the customer, and the value of 200 euros per month for each individual customer used in the calculation is a mean value based on the information from the target organization.

The costs related to ICT of tele-mediated solution can be divided to equipment and telecommunications costs. In the studied case, the telecommunications costs are included in the leasing price of the ICT equipment, which are the monthly fees of the customer terminals and the employee license for the software.

The calculation also aims to take into account the ICT costs related to the needed equipment and telecommunications start up, and costs related to the introduction of telerehabilitation practices. The calculation includes one-time purchase costs of the system and additional equipment such as needed headsets and web-cameras for the employees. Also training of the employees through user orientation to the ICT system used in the service is taken into account. The maintenance and support costs of the customer terminals are included in the leasing costs of the equipment and thus the maintenance and support costs include only the IT maintenance and support of target organization for the employee terminal.

The total cost of both methods based of the aforementioned data is presented on the bottom line of Table 1. The customer fees can be treated as an income for the municipality. With the aforementioned figures the calculation of costs already for one month favors the tele-homecare instead of traditional homecare. If the daily amount of visits in case of traditional homecare is decreased from the three visits used in the calculation to two visits, tele-homecare becomes profitable after two months.

Table 1. Calculation of costs related to traditional and tele-homecare. The figures are monthly costs, except for those that are marked with an asterisk $(*)$, which are one-time purchases. The figures are presented in euros

\begin{tabular}{|c|c|c|c|c|}
\hline \multirow[t]{2}{*}{ Cost categories } & \multicolumn{2}{|c|}{ Traditional homecare } & \multicolumn{2}{|c|}{ Tele-homecare } \\
\hline & Municipality & Customer & Municipality & Customer \\
\hline Work costs & 6469 & & 6469 & \\
\hline Travel costs & & & & \\
\hline - direct & 1935 & & & \\
\hline - work time used for travelling & 7824 & & & \\
\hline Client fees & & 5000 & & 4500 \\
\hline ICT costs & & & 1285 & \\
\hline $\begin{array}{l}\text { Costs related to introduction } \\
\text { of tele-homecare }\end{array}$ & & & & \\
\hline $\begin{array}{l}\text { - purchases and technical } \\
\text { realization }\end{array}$ & & & $455^{*}$ & \\
\hline - introduction & & & $5208 *$ & \\
\hline - user orientation & & & $647 *$ & \\
\hline - maintenance and support & & & 67 & \\
\hline Total cost & 16228 & 5000 & 14130 & 4500 \\
\hline Total cost for municipality & 11228 & & 9630 & \\
\hline
\end{tabular}

\section{DISCUSSION}

As a limitation for the above presented calculation it must be noticed that it is based on a single case in a single organization and thus the results are not necessarily generalizable to other contexts. In addition, approximations were used in several cost categories, due to the fact that not all figures were not obtainable at 
the time of the calculation. Most noticeable limitations of the presented calculation according to authors are following:

The costs related to the introduction of telerehabilitation practices are often hard to estimate, and thus are often omitted in calculations (McLean et al., 2013, Tousignant et al., 2015). These costs include definition and design, purchases and technical realization, introduction, user orientation and maintenance and support. In this case the definition of the need and design of the introduced system was realized over a longer time period as a part of normal development activities of the organization and the services it offers for its customers, and was thus not considered in the calculation. However, it can be estimated to constitute a significant figure. The calculation takes into account the purchases and technical realization, introduction, user orientation and maintenance of the ICT system. The considered user orientation, however, only covers the one-time initial user orientation at the start-up of the service and not the familiarization for upcoming employees.

In addition, the introduction of a new service takes time and the amount of customers is likely to increase gradually through the marketing and information shared by the organization to potential customers. The gradual increase of the amount of customers was not included in the calculation but instead an assumption of introduction of the service to 25 customers straight away was made. In reality finding the suitable customers calls for work on behalf of the employees in informing and advertising the service to potential customers as well as assessing their suitability for the service in terms of the evaluation of their performance ability.

As seen in the calculation, the travel costs in the traditional service are a dominant source of costs. These are highly dependent on the locations of the homes of the customers at one time and the routing of the employees visiting the customers. This therefore constitutes a significant source of uncertainty in the calculation through the used approximations. An additional bonus in favor of the introduction of telerehabilitation practices is that it also contributes to environmental sustainability by reducing carbon dioxide emissions through decreased need for travelling. Especially in today's situation that calls for actions that can have an effect on climate change this can be viewed as an important benefit.

The need for assistance in using the telerehabilitation equipment was also identified in earlier work (Kekkonen et al. 2019). An e-assistant that aids the client with the remote technology could be an employee of homecare or technical services, or even a family member. In our case, there was no identified need to an e-assistant other than the homecare service employee. Also identified in possible sources cost categories related to traditional rehabilitation is the loss of earnings on behalf of the customer in using the service due to the time reserved by the use. However, for the elderly customers the loss of earnings due to the use of health and welfare services is also not applicable.

The effectiveness of telerehabilitation as compared to traditional rehabilitation is an important issue to be considered. Obviously, the decision on choosing the appropriate service for each individual customer is important. This calls for the expertise of the rehabilitation professionals in terms of evaluating the performance ability of the potential customer. Also, in reality it might be that a hybrid solution including both tele- and traditional rehabilitation could be a suitable solution for many customers. In addition to the abilities and condition of the elderly customer, also the motivation of the customer to use the equipment (Early et al., 2017, McLean et al., 2013) and the needs for customer support and employee attitudes should be taken into consideration and studied further (McLean et al., 2013). These are aspects are considered in the on-going part of this project though the collection of experiences of both the customers and the employees during the introduction and early phases of telerehabilitation pilots, which will be reported later on.

The case studied in this article focuses on tele-homecare. Homecare cannot necessarily be considered rehabilitation, but it is an essential factor that has an effect on the performance ability of the elderly. Providing tele-homecare to those individuals that have the abilities that are needed for independent living, can aid them in retaining their performance ability in everyday life with a suitable amount of support through tele-homecare. This can be seen as a sign of social sustainability from the municipality to their inhabitants. 


\section{CONCLUSION}

During this project we aim to develop both cost-effective and high-quality telerehabilitation services for rural areas. Our aim is to provide analyses that could be used as community level information when decisions are to be made on rehabilitation and telerehabilitation practices at countryside. The results of the calculation presented in this article speak for the economic viability of introduction of tele-homecare solution. Next steps include further calculations and sensitivity analyses to the data as well as similar cost analyses for smaller-scale telerehabilitation practices. Qualitative data on the experiences of both customers and homecare employees is also gathered and analysed during the project to study the functionality and usability of the solution considering both the perspectives of customers and employees. These results are expected to shed light in the discussion of the quality and effectiveness of tele-homecare solution.

\section{ACKNOWLEDGEMENT}

The project is funded by the European Agricultural Fund for Rural Development.

\section{REFERENCES}

Dorsey, E. R. and Topol, E. J., 2020. Digital medicine - Telemedicine 2020 and the next decade. In The Lancet, Vol. 395, No. 10227 , pp. 859.

Early, F. et al, 2017. A Case Series of an Off-the-Shelf Online Health Resource with Integrated Nurse Coaching to Support Self-Management in COPD. InInternational Journal of COPD, Vol. 12, pp. 2955-2967.

Heymann, D.L. and Shindo, N., 2020. COVID-19: what is next for public health? In The Lancet, Vol. 395, No. 10224, pp. $542-545$

Hill, A., 2010. Telerehabilitation in Scotland: Current Initiatives and Recommendations for Future Development. In International Journal of Telerehabilitation, Vol. 2, No. 1, pp. 7-14.

Hollander, J.E. and Carr, B.G., 2020. Virtually perfcet? Telemedicine for Covid-19. In New England Journal of Medicine, DOI: 10.1056/NEJMp2003539.

Kekkonen, P. et al, 2019. Considerations on the Benefits of Using Telerehabilitation Practices in the Countryside. In: Patrone Cotrim, T., Serranheira, F., Sousa, P., Hignett, S., Albolino, S. \& Tartaglia, R. (eds). Building health and social care systems for the future: Demographic changes, digital age and human factors. 6th edition of the International Conference Healthcare Ergonomics and Patient Safety, 3-5 July 2019, Lisbon, Portugal, pp. 59-63.

McLean, S. et al, 2013. The Impact of Telehealthcare on the Quality and Safety of Care: A Systematic Overview. In PLoS ONE, Vol. 8, No. 8 pp. e71238.

Oxenburgh, M., and Marlow, P., 2005. The Productivity Assessment Tool: Computer-Based Cost Benefit Analysis Model for the Economic Assessment of Occupational Health and Safety Interventions in the Workplace. In Journal of Safety Research, Vol. 36, No. 3, pp. 209-214.

Selzler, A.-M. et al, 2018. Telehealth Pulmonary Rehabilitation: A Review of the Literature and an Example of a Nationwide Initiative to Improve the Accessibility of Pulmonary Rehabilitation. In Chronic Respiratory Disease, Vol. 15, No. 1, pp. 41-47.

Tousignant, M. et al, 2015. Cost Analysis of In-Home Telerehabilitation for Post-Knee Arthroplasty. In Journal of Medical Internet Research, Vol. 17, No. 3, pp. e83. 